\title{
LA RECHERCHE ARCHÉOLOGIQUE AU QUÉBEC. QUELLE PLACE AU SOLEIL ?
}

\author{
A pesquisa arqueológica no Quebec. \\ Qual lugar ao sol?
}

Pierre Desrosiers*

\begin{abstract}
RÉSUMÉ
Le Québec, comme toutes les provinces canadiennes, a légiféré sur la protection du patrimoine archéologique. Ce faisant, elle encadre du même coup la recherche. Comment s'harmonise alors la recherche avec la pratique actuelle de l'archéologie ? La législation permet-elle de mener à terme la recherche archéologique afin que le citoyen puisse bénéficier des connaissances acquises ? Le survol met en scène les divers acteurs associés à la recherche archéologique au Québec et permet de faire des constats alarmants sur la production de la connaissance. Celle-ci est bel et bien reléguée au second plan, derrière la protection du patrimoine archéologique. N'empêche que la finalité de la protection demeure, à ce jour, ambiguë.
\end{abstract}

Mots-clés: archéologie, loi, recherche, protection, patrimoine

\begin{abstract}
Quebec, like all Canadian provinces, has legislated for the protection of archaeological heritage. In so doing, it also guides archaeological research. How does the research fit into the current practice of archeology? Does the legislation allow for archaeological research to be conducted in such a way that citizens can benefit from the knowledge acquired? This overview of the various actors associated with archaeological research in

* Chercheur adjoint au (pesquisador adjunto) CELAT. 1030, Ave des SciencesHumaines, Université Laval, Québec, G1V 0A6 - CANADA.

E-mail: desrosiers.pierre896@videotron.ca
\end{abstract}


Quebec exposes some rather alarming findings on knowledge production. Knowledge production is well and truly relegated to the background, after the protection of archaeological heritage. The purpose of heritage protection remains, therefore, ambiguous.

Keywords: Archaeology, legislation, research, protection, heritage

\section{RESUMO}

O Quebec, assim como todas as províncias canadenses, legislou sobre a proteção do patrimônio arqueológico. Em consequência, a pesquisa também ficou enquadrada por esta lei. Neste contexto, de que forma a pesquisa e a prática atual da arqueologia se harmonizam? Até onde a legislação permite desenvolver a pesquisa arqueológica para que o cidadão possa beneficiar-se do conhecimento resultante das pesquisas? Este texto apresenta um panorama dos diferentes atores associados à pesquisa arqueológica no Quebec e faz constatações alarmantes sobre a produção de conhecimento. A pesquisa está realmente relegada para o segundo plano, após a proteção do patrimônio arqueológico. Não obstante, a finalidade da proteção permaneça até hoje ambígua.

Palavras-chave: arqueologia, lei, pesquisa, proteção, patrimônio

\section{Introduction}

La recherche est au cœur de la pratique archéologique ; sans elle il n'y a pas d'acquisition de nouvelles connaissances, c'est la stagnation intellectuelle. Le dictionnaire Larousse propose une définition de l'archéologie scientifique qui s'applique encore aujourd'hui : « $\mathrm{Au} \mathrm{XX}$ e siècle, l'archéologie devient une discipline à part entière, soutenue par une législation et un enseignement. Le travail archéologique comprend quatre stades principaux: la prospection, la fouille, l'étude du matériel qui mène à son interprétation et, enfin, la publication ». Le présent exercice explore la notion de recherche archéologique dans la législation québécoise, ontarienne et française, avant de s'attarder sur qui fait de la recherche 
archéologique et dans quels contextes elle se concrétise au Québec en 2013 et 2014.

Comme point de départ, il faut revenir à la notion de fouille. Sans elle, il n'y a pas de recherche scientifique possible. Ce qui se fait préalablement sur le terrain, l'inventaire d'un territoire ou l'évaluation d'un site, ce qu'on peut appeler de la prospection, ne sont que des étapes préparatoires à la recherche. La fouille est l'essence même du travail de l'archéologue fondé sur l'étude du contexte dans lequel les vestiges mobiliers et immobiliers sont mis au jour. C'est en vue de la fouille qu'une problématique, des objectifs et une stratégie de recherche sont déterminés ; c'est ce qui oriente la cueillette de données en vue des analyses qui seront effectuées ultérieurement en laboratoire, de l'interprétation des résultats et de la diffusion des connaissances qui s'en suivront.

Les connaissances acquises par la fouille et les analyses permettent de reconstituer le passé d'un site, l'histoire de ses occupants et leurs modes de vie. À vrai dire, sans fouille il est possible de faire des recherches scientifiques, mais l'essence même de la recherche archéologique réside dans la reconstitution du contexte synchronique et diachronique du passé des êtres humains qui nous ont précédés sur le territoire.

\section{Le cadre légal entourant la recherche archéologique}

Le cadre légal varie d'une juridiction à l'autre ; cela peut être dû à bon nombre de facteurs dont la tradition, les pressions sociales, les groupes d'intérêts, etc. Le Québec est une province canadienne et son héritage français oriente et définit plusieurs des actions gouvernementales en matière d'archéologie alors que les autres provinces canadiennes, comme l'Ontario, possèdent un héritage en grande partie anglo-saxon qui, lui aussi, influence les actions gouvernementales en matière d'archéologie. L'archéologie française 
sert ici à comparer une structure gouvernementale européenne par rapport à une structure nord-américaine.

\subsection{La législation québécoise}

La loi sur le patrimoine culturel (2012) $)^{1}$ et le règlement sur la recherche archéologique $(2013)^{2}$ réfèrent nommément à la recherche archéologique et y associent les fouilles et les relevés sans toutefois expliquer ce qu'elle signifie. La législation fixe des balises générales pour l'obtention d'un permis de recherche archéologique: le requérant et son équipe doivent présenter leurs qualifications, ce qui inclut leur formation, leurs expériences professionnelles, leurs publications et les organismes auxquels ils se rattachent. Il revient alors au ministère de la Culture et des Communications d'évaluer si les informations fournies garantissent « l'exécution satisfaisante et complète du projet » (art. 69). La législation n'exclut pas spécifiquement les amateurs, mais ces derniers ne peuvent faire une demande s'ils n'ont pas la formation et l'expérience professionnelles requises. Enfin, en vertu du Guide intérimaire en matière de consultation des communautés autochtones $(2008)^{3}$, le ministère doit évaluer si l'intervention archéologique peut porter atteinte aux droits revendiqués par une ou des communautés autochtones ; cela se traduit par une consultation préalable auprès d'elles ${ }^{4}$.

Les différences entre les dispositions réglementaires de 1973 et de 2013 sont importantes sur le plan scientifique. Celles de 1973 s'appuyaient sur un programme de recherche comprenant la nature de la recherche et la problématique envisagée, ainsi qu'un programme de diffusion et de mise en valeur. Lesdits programmes ne s'avèrent plus pertinents en 2013. Sauf qu'en procédant ainsi, la démarche archéologique n'est plus aussi cohérente, ni aussi explicite sur le plan scientifique. Le résultat fait qu'on dissocie l'intervention

\footnotetext{
1http://legisquebec.gouv.qc.ca/fr/ShowDoc/cs/P-9.002

2http://legisquebec.gouv.qc.ca/fr/ShowDoc/cr/P-9.002,\%20r.\%202.1

3https://www.autochtones.gouv.qc.ca/publications_documentation/publications/guide_i nter_2008.pdf

4 La consultation auprès des communautés autochtones a débuté en 2015.
} 
archéologique d'une démarche globale d'acquisition de connaissance, ce qui rend l'intervention archéologique plus technique.

\subsection{La législation ontarienne}

La législation ontarienne est plus étoffée en matière d'archéologie que celle du Québec. D'abord, il faut obtenir une licence pour pratiquer l'archéologie en Ontario. Celle-ci est valide pour une période d'un à trois ans, ce qui permet à son détenteur de réaliser des interventions archéologiques sur le territoire en fonction de différents stades (1 à 4) de complexité du travail de terrain. Ces stades permettent aux archéologues les plus qualifiés de réaliser des fouilles et aux amateurs de contribuer à la recherche sur le terrain en réalisant des activités complémentaires (par exemple, la cueillette d'artefacts sur un site).

La législation est orientée essentiellement vers la gestion et la protection du patrimoine archéologique ; les interventions archéologiques qu'elle autorise y sont assujetties. Le volumineux cahier (193 pages) des Normes et Directives à l'intention des archéologues-conseils $(2011)^{5}$ présente des balises strictes et détermine ce qu'il faut faire en fonction des quatre catégories de licences ainsi que dans le contexte de l'aménagement du territoire. Même les conditions de fouilles sont déterminées en fonction de l'aménagement $\mathrm{du}$ territoire et, en cela, rejoint une autre loi ontarienne, la Loi sur l'aménagement $d u$ territoire $(1990)^{6}$ où l'archéologie joue un rôle majeur. Enfin, une consultation des nations autochtones s'impose lorsqu'une intervention archéologique peut avoir «des effets indésirables sur les droits ancestraux ou issus de traités, établis ou affirmés ${ }^{7}$.

La recherche est à peine mentionnée dans la législation ontarienne. De fait, il existe une licence de recherche appliquée pour les chercheurs universitaires mais, globalement, la reconnaissance gouvernementale de l'expertise archéologique se limite à la

5http://www.mtc.gov.on.ca/fr/archaeology/archaeology_s_g.shtml $6 \mathrm{http} / / /$ legisquebec.gouv.qc.ca/fr/ShowDoc/cs/A-19.1

ontario

7 https://www.ontario.ca/fr/page/obligation-de-consulter-les-peuples-autochtones-en- 
délivrance d'une licence professionnelle. Non seulement l'archéologue doit-il être en possession d'une maîtrise en archéologie, mais il doit aussi faire partie d'une association d'archéologues avec un code de déontologie. À cela s'ajoute l'expérience démontrée de pratique professionnelle sur le terrain, en matière de gestion et de supervision et, finalement, en rédaction de rapports.

En somme, l'État ontarien concentre son action sur la bonne pratique professionnelle, sans toutefois accorder d'intérêt particulier à l'acquisition et à la diffusion des connaissances. Celles-ci peuvent évidemment être acquises et diffusées dans le cadre législatif ontarien, mais ce n'est pas le but visé. Il en va de même pour le traitement ambigu des collections archéologiques issues des interventions de terrain ; celles-ci se retrouvent chez les archéologues considérés comme étant les gardiens des biens de l'État.

\subsection{L'archéologie préventive en France}

En France, l'archéologie s'inscrit dans un processus légal qui remonte à la loi Carcopino du 27 septembre $1941^{8}$ réglementant les fouilles archéologiques. Pour leur part, le Code du patrimoine adopté en $2000^{9}$, puis la Loi du 17 janvier $2001^{10}$ relative à l'archéologie préventive, ouvrent la voie à la création de l'Institut national de recherches archéologiques préventives (INRAP) avec un mandat ambitieux :

L'archéologie préventive, qui relève de missions de service public, est partie intégrante de l'archéologie. Elle est régie par les principes applicables à toute recherche scientifique. Elle a pour objet d'assurer, à terre et sous les eaux, dans les délais appropriés, la détection, la conservation ou la sauvegarde par l'étude scientifique des éléments du patrimoine archéologique affectés ou

\footnotetext{
8 https://www.legifrance.gouv.fr/affichTexte.do?cidTexte=LEGITEXT000006074257.

9https://www.legifrance.gouv.fr/affichCodeArticle.do?cidTexte=LEGITEXT00000607 4236\&idArticle=LEGIARTI000006845683.

10https://www.legifrance.gouv.fr/affichTexte.do?cidTexte=JORFTEXT000000221337 $\&$ dateTexte $=\&$ categorieLien $=$ id.
} 
susceptibles d'être affectés par les travaux publics ou privés concourant à l'aménagement. Elle a également pour objet l'interprétation et la diffusion des résultats obtenus. (CODE DU PATRIMOINE, art. L-521-1)

L'approche préventive privilégiée par cette entité gouvernementale conserve une logique propre à la démarche archéologique qui se prolonge dans l'analyse, l'interprétation des résultats et leur diffusion: l'acquisition et la diffusion des connaissances paraissent ici bien incarnées. De plus, l'approche préventive se veut soucieuse de gérer et de protéger le patrimoine archéologique et inscrit son action dans un cadre de développement social et économique, ce qui est un défi considérable en soi.

La recherche semble être le moteur qui anime l'approche préventive préconisée par l'État français et elle ne semble avoir été ni réduite, ni altérée, face au mandat de l'INRAP, même par rapport à d'autres institutions nationales tel que l'Institut national de la recherche scientifique (INRS).

\subsection{Résumé}

Trois juridictions, trois visions différentes, malgré un dénominateur commun: l'encadrement légal de la pratique archéologique de terrain. Toutes trois reconnaissent le besoin de légiférer pour protéger ce patrimoine culturel, mais les façons de faire divergent considérablement, notamment sur le plan de la gestion, de la recherche, de la diffusion et de l'intégration du patrimoine archéologique au milieu de vie.

L'attention portée à la recherche archéologique - base de toute formation universitaire en archéologie - est très variable d'une juridiction à l'autre. À ce titre, il vaut la peine de réitérer qu'au Québec la formation universitaire est un élément considéré et évalué dans toute demande de permis, alors qu'en Ontario c'est un prérequis et qu'en France c'est la base de toute recherche scientifique. En ce qui a trait à la notion de recherche proprement dite, elle demeure floue au Québec, alors qu'elle est appliquée à l'aménagement du territoire en 
Ontario et fermement enchâssée dans une archéologie préventive en France.

Le lien entre les pratiques de terrain et leur insertion dans une démarche archéologique aboutie, c'est-à-dire qui permet d'acquérir et de diffuser des nouvelles connaissances, semble implicite dans les législations canadiennes alors qu'il est explicite en France. En fait, les législations canadiennes ont tendance à se restreindre aux pratiques de terrain sans aborder le contexte plus global de la démarche archéologique tandis qu'il est expressément reconnu dans la législation française. Ce faisant, les premières introduisent une nouvelle finalité : la protection du patrimoine archéologique qui se traduit généralement par la levée de la contrainte archéologique sous différentes formes : des interventions archéologiques, l'évitement et la protection à long terme. L'absence de lien entre la protection, la recherche et la diffusion a ici l'effet de compartimenter les activités archéologiques alors qu'elles devraient s'inscrire dans un processus global et continu.

\section{Qui fait de la recherche archéologique au Québec?}

Le tour d'horizon qui suit couvre plus spécifiquement la réalité autour de la recherche archéologique des années 2013 à 2015.

\subsection{Les universités}

On ne peut aborder la recherche archéologique sans tenir compte tout d'abord de la production universitaire. À cet égard, l'offre universitaire en matière de formation archéologique est fort riche (figure 1). 
Figure 1 : Les universités du Québec offrant une formation en archéologie.

\begin{tabular}{|c|c|c|}
\hline Université & Département & $\begin{array}{l}\text { Programmes / cours offerts en } \\
\text { archéologie }\end{array}$ \\
\hline Université Laval & $\begin{array}{l}\text { Département des } \\
\text { sciences historiques }\end{array}$ & $\begin{array}{l}\text { Programmes en archéologie } \\
\text { offerts aux étudiants au } \\
\text { baccalauréat (premier cycle), à la } \\
\text { maîtrise et aux études doctorales. }\end{array}$ \\
\hline $\begin{array}{l}\text { Université de } \\
\text { Montréal }\end{array}$ & $\begin{array}{l}\text { Département } \\
\text { d'anthropologie }\end{array}$ & $\begin{array}{l}\text { Programmes en archéologie } \\
\text { offerts aux étudiants au } \\
\text { baccalauréat (premier cycle), à la } \\
\text { maîtrise et aux études doctorales. }\end{array}$ \\
\hline $\begin{array}{l}\text { Université } \\
\text { McGill }\end{array}$ & $\begin{array}{l}\text { Département } \\
\text { d'anthropologie }\end{array}$ & $\begin{array}{l}\text { Programmes en archéologie } \\
\text { offerts aux étudiants au } \\
\text { baccalauréat (premier cycle), à la } \\
\text { maîtrise et aux études doctorales. }\end{array}$ \\
\hline $\begin{array}{l}\text { Université du } \\
\text { Québec à } \\
\text { Montréal }\end{array}$ & $\begin{array}{l}\text { Département d'histoire } \\
\text { de l'art }\end{array}$ & Cours seulement \\
\hline $\begin{array}{l}\text { Université du } \\
\text { Québec à } \\
\text { Chicoutimi }\end{array}$ & Département d'histoire & $\begin{array}{l}\text { Certificat au niveau du } \\
\text { baccalauréat (premier cycle) en } \\
\text { archéologie }\end{array}$ \\
\hline $\begin{array}{l}\text { Université du } \\
\text { Québec à } \\
\text { Rimouski }\end{array}$ & $\begin{array}{l}\text { Département d'histoire } \\
\text { régionale }\end{array}$ & Cours seulement \\
\hline
\end{tabular}

En milieu universitaire, la recherche se conjugue avec la formation. En matière d'archéologie québécoise ${ }^{11}$, celle-ci couvre à des degrés divers les aspects traditionnels de la discipline représentatifs des cultures autochtones (archéologie préhistorique) et eurocanadiennes (archéologie historique) ; elle explore de façon substantielle la culture matérielle (artéfacts) prélevée sur les sites, ainsi que les écofacts qui leur sont associés pour mieux reconstituer l'environnement culturel et naturel de l'occupation humaine; elle se fait aussi avec des collections de référence pour mener des études spécialisées sur divers matériaux trouvés sur les sites du Québec ; elle développe des approches statistiques et archéométriques ; elle utilise des supports géomatiques et des logiciels de modélisation ; et, enfin, elle n'hésite pas à développer des partenariats avec d'autres

11 L'archéologie classique est enseignée également dans les universités québécoises, sauf que les deux archéologies ne se retrouvent pas dans les mêmes départements. 
laboratoires scientifiques universitaires en archéologie et dans plusieurs domaines connexes. La formation universitaire offerte au Québec est de calibre international grâce aux chercheurs qui y travaillent, aux installations et équipements disponibles, et à l'encadrement et à la synergie qui s'en suivent entre les professeurs, les étudiants et la communauté des archéologues en général.

En plus d'une formation théorique, l'université offre des stages pratiques. En 2015, toutes les universités québécoises possédaient un chantier-école et parfois même plusieurs (figure 2). Le chantier-école se déroule sur un site qui permet généralement de construire un programme de recherches à long terme et de faire des fouilles pendant plusieurs années.

Figure 2 : Les chantiers-écoles universitaires au Québec en 2015.

\begin{tabular}{|c|c|}
\hline \multicolumn{2}{|c|}{ Les chantiers-écoles en archéologie au Québec en 2015} \\
\hline Universités - Collèges & Chantiers-écoles \\
\hline Université de Montréal & $\begin{array}{l}\text { - Site Droulers-Tsiionhiakwatha (village iroquoien), Saint- } \\
\text { Anicet, Montérégie } \\
\text { - Site Kruger, Sherbrooke (site amérindien de la période } \\
\text { Archaïque) } \\
\text { - Ferme du Mont-Royal, Montréal (site euroquébécois?) } \\
\text { - Île Saint-Bernard (site amérindien et euroquébécois?) }\end{array}$ \\
\hline Université Laval & $\begin{array}{l}\text { - Îlot des Palais (palais de l'intendant, archéologie urbaine), } \\
\text { Québec } \\
\text { - Fort Saint-Jean (militaire), Saint-Jean, Montérégie }\end{array}$ \\
\hline $\begin{array}{l}\text { Université du Québec à } \\
\text { Chicoutimi }\end{array}$ & - Baie-Sainte-Marguerite (préhistoire régionale), Saguenay \\
\hline $\begin{array}{l}\text { Université du Québec à } \\
\text { Rimouski }\end{array}$ & $\begin{array}{l}\text { - Camp forestier de la Matapédia (histoire régionale), } \\
\text { Amqui, Bas-Saint-Laurent }\end{array}$ \\
\hline
\end{tabular}

Les universités publient les résultats des recherches archéologiques faites au Québec, directement ou indirectement. À l'Université Laval, par exemple, le Centre de recherche CulturesArts-Sociétés (CELAT), qui regroupe des chercheurs de l'Université Laval, de l'Université du Québec à Montréal et de l'Université du Québec à Chicoutimi, publie les recherches archéologiques des chercheurs. Les Cahiers du CELAT sont d'ailleurs privilégiés pour diffuser les résultats des fouilles menées sur le site du Palais de l'intendant à Québec ; la dernière étant celle de Simoneau (2014). Les Presses de l'Université Laval publient parfois des recherches 
archéologiques ; la dernière en lice est celle de Desrosiers (2011). Par contre, les Presses de l'Université du Québec et les Presses de l'Université de Montréal n'ont pas publié d'ouvrages archéologiques depuis plusieurs années. Les chercheurs ont alors recours aux maisons d'édition intéressées à publier les résultats de leurs recherches (voir plus loin).

\subsection{Les institutions gouvernementales}

$\mathrm{Au}$ ministère de la Culture et des Communications du Québec (MCC), il ne se fait plus de recherche archéologique depuis plusieurs décennies, ni sur le terrain, ni au laboratoire et à la réserve d'archéologie du Québec, le dépôt gouvernemental des collections archéologiques du Québec. C'est principalement via les ententes de développement culturel avec les municipalités que la recherche se fait lorsque celles-ci veulent bien en faire car ce sont elles qui orientent leurs choix en fonction de leurs besoins en matière de connaissance, de conservation ou d'aménagement du territoire (voir plus loin).

D'autres ministères et sociétés d'État tels que le ministère des Transports du Québec, Hydro-Québec ou la Commission de la Capitale nationale de Québec investissent des sommes importantes pour prendre en compte le patrimoine archéologique. Leurs projets se font en fonction du respect des lois qui assurent la protection du patrimoine archéologique au Québec.

$\mathrm{Au}$ sein du gouvernement fédéral, la majorité des projets archéologiques sont réalisés sous la gouverne de l'agence Parcs Canada dans les lieux historiques et les parcs nationaux du Canada. Depuis 2014, le gouvernement fédéral réinvestit massivement dans les projets de restauration des bâtiments historiques où le patrimoine archéologique est pris en compte et des interventions archéologiques sont faites en conséquence (PARCS CANADA, 2014). En matière de recherche, il ne reste que l'expertise en archéologie subaquatique qui, depuis Ottawa, s'investit régulièrement dans des projets au Québec.

\subsection{Les instances municipales}

Les villes de Montréal et de Québec disposent annuellement d'un budget de plusieurs centaines de milliers de dollars pour réaliser 
des projets en archéologie. Les subventions accordées par le MCC en vertu des ententes de développement culturel sont paritaires, c'est-àdire que chaque partenaire défraie le même montant annuellement, soit $50 \%$ du total. Les projets sont généralement associés à la qualité de vie des citoyens en milieu urbain, à la protection et à la mise en valeur du patrimoine culturel qui bénéficie d'une protection légale, soit les sites patrimoniaux déclarés ou classés et les immeubles classés. Le patrimoine archéologique est pris en considération dans cette vision globale de la protection du patrimoine culturel. Pour que des fouilles soient réalisées, elles doivent habituellement être encadrées par un projet qui répond à des besoins d'aménagement urbain : parcs, lieux patrimoniaux, etc. La diffusion de la recherche archéologique municipale se fait essentiellement via leur site web ou, encore, dans les Cahiers du CELAT à Québec et dans la collection Patrimoine archéologique de Montréal à Montréal.

Les autres municipalités locales et régionales du Québec ne bénéficient que d'une maigre subvention en patrimoine dans le cadre de leurs ententes de développement culturel ; néanmoins plusieurs voient les recherches archéologiques comme une valeur ajoutée à la qualité de vie de leurs citoyens et réussissent à diffuser les connaissances acquises.

\subsection{Les organismes autochtones}

Certains organismes autochtones bénéficient d'ententes depuis des décennies avec d'autres partenaires gouvernementaux fédéraux et provinciaux pour réaliser des projets culturels. C'est le cas de l'Administration régionale Crie ${ }^{12}$ et de l'Institut culturel Avataq ${ }^{13}$. Ces mêmes organismes comptent des archéologues ${ }^{14}$ dans leurs effectifs et dirigent des interventions archéologiques dans les communautés autochtones; ces interventions servent autant à la recherche qu'à des fins de sensibilisation et d'éducation, en particulier auprès des jeunes. Ils bénéficient de laboratoires pour la

12 Administration régionale Crie www.gcc.ca.

13 Institut culturel Avataq : www.avataq.qc.ca.

14 Des coupures budgétaires à l'automne 2015 ont cependant entraîné le départ de la majorité de l'équipe d'archéologues au sein de l'Institut culturel Avataq. 
poursuite de recherches sur le terrain et de réserves pour conserver leurs collections archéologiques. De plus, les Cris ont maintenant un musée qui présente les résultats des recherches archéologiques.

\subsection{Les institutions muséales}

Plusieurs institutions muséales sont subventionnées par les paliers municipaux et provinciaux et certains de leurs projets présentent des volets archéologiques. Le projet de recherche le plus important des dernières années, piloté par le musée de Pointe-àCallière, concerne la Cité d'archéologie et d'histoire de Montréal où des travaux majeurs ont permis de dégager plusieurs vestiges archéologiques enfouis dans le sol à des fins de mise en valeur. Pointe-à-Callière produit aussi des ouvrages de référence en archéologie au Québec, en collaboration avec les Éditions de l'Homme : deux ont déjà vu le jour Air (PINTAL et al., 2015) et Fragments d'humanité (POTHIER, 2016), et trois autres (Eau, Terre et $\mathrm{Feu}$ ) sont en voie de rédaction.

\subsection{Les organismes nationaux}

L'Association des archéologues du Québec encourage la recherche archéologique via son colloque annuel et ses publications, dont la revue scientifique Archéologiques ${ }^{15}$. Rédigé au moment de la création de l'association en 1979, le Code d'éthique et des normes professionnelles $^{16}$ énonce les responsabilités de l'archéologue envers ses collègues et les communautés en matière de recherche ; il affirme que : "L'archéologue est responsable de la planification et/ou de la réalisation de recherches archéologiques puis de la diffusion de leurs résultats " (point 3). Le code correspond essentiellement à l'esprit d'autres codes de déontologie ou de conduite rédigés par des organismes nord-américains qui, comme le souligne Wylie (2003, p. 25), ont pour but de permettre aux archéologues d'harmoniser les

15 La revue Archéologiques : http://www.archeologie.qc.ca/publications/.

16 Le code d'éthique de l'Association des archéologues du Québec: http://www.archeologie.qc.ca/lassociation/code-dethique/. 
objectifs spécialisés de leur profession avec les enjeux de société dans lesquelles ils vivent et pratiquent leur profession.

De son côté, Archéo-Québec ${ }^{17}$, l'organisme panquébécois réseau de la diffusion de l'archéologie, ne mène pas de recherche archéologique. Son mandat l'amène plutôt à organiser annuellement le Mois de l'archéologie et à collaborer avec un vaste réseau d'organismes provenant de divers milieux qui s'intéressent à l'archéologie. Ses activités s'étendent aussi à la rédaction de divers documents destinés aux municipalités, au tourisme régional et à la diffusion du patrimoine archéologique.

\subsection{Les éditeurs de périodiques et les maisons d'édition}

Trois périodiques scientifiques diffusent la recherche archéologique qui se fait au Québec: Archéologiques, Recherches amérindiennes au Québec ${ }^{18}$ et Études Inuit Studies ${ }^{19}$. La Société Recherches amérindiennes au Québec dispose aussi de collections dont Paléo-Québec - qui permettent de publier des ouvrages scientifiques en archéologie, le dernier en lice étant le collectif d'auteurs dirigé par Claude Chapdelaine sur le village iroquoien Mailhot-Curran en Montérégie (2015). Pour sa part, la revue Études Inuit Studies a publié en 2015 un article sur les recherches menées par l'Institut culturel Avataq (DESROSIERS et RAHM, 2015).

Les maisons d'édition québécoises publient peu souvent des ouvrages à contenu archéologique. Néanmoins, l'ouvrage Archéologie de l'Amérique coloniale française, qui dépasse largement les frontières québécoises tout en lui faisant jouer un rôle central, a été publié récemment par Lévesque éditeur (MOUSSETTE et WASELKOV, 2013). Comme mentionné plus tôt, les Éditions de l'Homme a publié en 2015 et 2016 deux collectifs d'auteurs réalisés par Pointe-à-Callière, le musée d'archéologie et d'histoire de Montréal (Air et Fragments d'humanité).

\footnotetext{
17 Le réseau Archéo-Québec : www.archeoquebec.com.

18 La revue Recherches amérindiennes au Québec: http://recherchesamerindiennes.qc.ca/site/.

19 La revue Études Inuit Studies : https://www.etudes-inuit-studies.ulaval.ca/.
} 
Encore plus rares sont les recherches archéologiques québécoises publiées ailleurs au Canada, exception faite de la collection Mercure rattachée maintenant aux Presses de l'Université d'Ottawa et bénéficiant d'une subvention du ministère du Patrimoine canadien. La dernière en lice est celle de Bibeau et al. (2015), une publication des recherches menées sur le territoire cri et subventionnées par Hydro-Québec dans le cadre de l'aménagement hydroélectrique de l'Eastmain-1. Quant au Musée canadien de l'histoire, auparavant le Musée canadien des civilisations, il a publié, en collaboration avec les Presses de l'Université d'Ottawa, les recherches sur l'occupation iroquoienne à l'embouchure de la rivière Saguenay (PLOURDE, 2013).

\subsection{Résumé}

Ce tour d'horizon témoigne bien du fait que la recherche archéologique demeure au cœur des préoccupations des archéologues québécois, même s'ils n'ont pas toujours l'opportunité de la mener à terme.

Les chercheurs universitaires doivent composer avec la formation dans les chantiers-écoles et les laboratoires qu'ils gèrent, ce qui reporte d'autant les résultats de recherche ; la méthodologie scientifique prend ici le pas sur l'interprétation. Néanmoins, bon an, mal an, les publications scientifiques sont réalisées, sous forme de collectifs d'auteurs généralement. En dehors du monde universitaire, les institutions gouvernementales et municipales, ainsi que les institutions muséales et les organismes autochtones font elles aussi des recherches archéologiques, sauf que celles-ci sont subordonnées à des projets où l'archéologie demeure une composante parmi d'autres $\mathrm{du}$ projet d'aménagement, de développement, de sensibilisation, d'éducation ou de mise en valeur. Les fouilles réalisées dans un tel cadre font souvent l'objet d'analyses et aboutissent à des résultats probants. Elles sont toutefois moins fréquentes et les interprétations qui en résultent peinent à être publiées. Somme toute, les connaissances acquises au fil des ans trouvent un exutoire fort intéressant dans des collectifs d'auteurs destinés au grand public et présentés comme des livres-synthèses tels qu'Air (PINTAL et al. 2015) et Fragments d'humanité (POTHIER, 2016). 


\section{Qu'est-ce qui se fait comme recherche archéologique sur le terrain?}

Après avoir fait un tour d'horizon sur ceux qui font de la recherche et sur leurs publications, voici ce qui s'est fait comme fouilles archéologiques au Québec durant les années 2013 et 2014, le but étant ici de mieux saisir les contextes qui les ont motivés et les objectifs poursuivis. Ont été retenues les données disponibles sur les interventions archéologiques réalisées au Québec, toutes juridictions confondues $^{20}$. Plutôt que de porter sur l'ensemble des interventions (fouilles, inventaires, surveillances, etc.), ce qui a déjà fait l'objet d'un article (DESROSIERS, 2016), l'exercice se concentre sur les fouilles qui ont été réalisées durant cette période. Sont exclues les fouilles ouvertes au public, plutôt considérées comme des activités où la recherche n'est pas l'objectif premier. Sont inclus toutefois les chantiers-écoles, les fouilles proprement dites et les « divers » (figure 3 ). Cette dernière catégorie couvre un ensemble d'interventions au sein d'un même projet soumis au MCC en vue de l'obtention d'un permis de recherche archéologique ${ }^{21}$. Elle comprend la prospection, la fouille et la surveillance sur un site ou un territoire voué à une destruction imminente pour faire place à un aménagement ou à un projet de construction.

20 Les données proviennent du ministère de la Culture et des Communications via la Loi sur l'accès à l'information, ainsi que de Parcs Canada et d'autres organismes via des contacts individuels.

21 Cette catégorie reflète une réalité de la pratique archéologique au Québec où, pour éviter d'avoir à modifier son permis en cours de route, l'archéologue préfère énumérer dans sa demande initiale l'ensemble des interventions qui pourraient être réalisées. 
Figure 3 : Description sommaire des fouilles réalisées au Québec en 2013 et 2014.

\begin{tabular}{|c|c|}
\hline $\begin{array}{c}\mathbf{2 0 1 3} \\
28 \text { projets sur } 181(15 \%)\end{array}$ & $\begin{array}{l}\mathbf{2 0 1 4} \\
42 \text { projets sur } 216(19 \%)\end{array}$ \\
\hline $\begin{array}{l}\text { Nature des interventions : } \\
\text { - } \quad 16 \text { fouilles, } \\
\text { - } \quad 7 \text { chantiers-écoles, } \\
\text { - } \quad 5 \text { divers. } \\
\text { Contextes : } \\
\text { - } \quad 22 \text { projets culturels, } \\
\text { - } \quad 4 \text { projets de développement, } \\
\text { - } \quad 2 \text { projets d'aménagement urbain. }\end{array}$ & $\begin{array}{l}\text { Nature des interventions : } \\
\text { - } \quad 19 \text { fouilles, } \\
\text { - } \quad 16 \text { divers, } \\
\text { - } \quad 7 \text { chantiers-écoles. } \\
\text { Contextes : } \\
\text { - } \quad 26 \text { projets culturels, } \\
\text { - } \quad 10 \text { projets de développement, } \\
\text { - } \quad 6 \text { projets d'aménagement urbain. }\end{array}$ \\
\hline
\end{tabular}

À priori, moins de $20 \%$ des interventions archéologiques réalisées au Québec en 2013 et 2014 incluaient des fouilles, les autres interventions étant destinées principalement à la prospection (inventaires ou évaluations), à la surveillance lors des travaux d'excavation dans le cadre de projets de développement ou d'aménagement, ou à des activités destinées au public. Quant aux contextes, les projets culturels dominent largement d'une année à l'autre. Rappelons cependant que les obligations légales contribuent au nombre élevé de projets culturels qui sont généralement situés dans les sites patrimoniaux déclarés ou classés, en particulier à Québec et à Montréal, ou, encore, dans les lieux historiques nationaux du Canada. Les fouilles réalisées dans un tel contexte ne le sont pas nécessairement à des fins de recherche ; elles servent souvent à documenter un vestige et à libérer la contrainte archéologique en vue d'une mise en valeur, d'un projet de construction ou d'un aménagement urbain.

Les chantiers-écoles sont principalement menés par des universités et par des collèges. Les chantiers-écoles universitaires disposent de laboratoires et d'infrastructures d'envergure, ainsi que de conditions plus propices pour poursuivre les analyses, interpréter les résultats et publier le tout. En somme, à travers leur formation, les universités continuent à faire valoir la démarche traditionnelle d'acquisition et de diffusion des connaissances propre à la recherche archéologique.

Les projets divers où un ensemble d'interventions est prévu d'avance, répondent généralement à des obligations légales, contractuelles, ou les deux. Cette catégorie vise à documenter le 
patrimoine archéologique qui s'y trouve et à libérer la contrainte archéologique, c'est-à-dire à faire les interventions nécessaires pour que le promoteur puisse ensuite réaliser son projet en conformité avec la loi.

En associant les fouilles menées en 2013 et 2014 avec une problématique de recherche conformément à toute recherche archéologique traditionnelle, il s'avère que cette préoccupation scientifique est de moins en moins présente (figure 4).

Figure 4 : Les fouilles réalisées dans un contexte de recherche.

\begin{tabular}{|c|c|c|}
\hline \multicolumn{3}{|c|}{ Fouilles archéologiques au Québec (2013-2014) : Recherche } \\
\hline$\underline{\underline{2013}}$ & $\underline{\underline{2014}}$ & Commentaires \\
\hline Total de 181 projets & Total de 216 projets & $\begin{array}{lll}\begin{array}{l}\text { Augmentation } \\
\text { projets }(19 \%)\end{array} & \text { de } 35 \\
\end{array}$ \\
\hline $\begin{array}{l}38 \text { fouilles prévues }(20 \\
\%)\end{array}$ & $\begin{array}{l}48 \text { fouilles prévues (22 } \\
\%)\end{array}$ & $\begin{array}{l}\text { Faible augmentation de } \\
\text { fouilles en } 2014(2 \%) \text {. }\end{array}$ \\
\hline $\begin{array}{l}28 \text { fouilles réalisées (15 } \\
\%) \\
\bullet \quad 11 \text { avec } \\
\text { objectif de recherche } \\
\text { (dont } 7 \text { chantiers- } \\
\text { écoles), } \\
\bullet \quad 9 \text { avec pour } \\
\text { objectif de dégager et } \\
\text { documenter les vestiges, } \\
\text { - } \quad 8 \text { sans } \\
\text { problématique } \\
\text { particulière (potentiel } \\
\text { inconnu). }\end{array}$ & $\begin{array}{l}42 \text { fouilles réalisées (19 } \\
\% \text { ) } \\
\text { • } \quad 20 \text { avec } \\
\text { objectif de recherche } \\
\text { (dont } 7 \text { chantiers- } \\
\text { écoles), } \\
\text { - } \quad 16 \text { avec pour } \\
\text { objectif de dégager et } \\
\text { documenter les } \\
\text { vestiges, } \\
\text { - } \quad 6 \text { sans } \\
\text { problématique } \\
\text { particulière (potentiel } \\
\text { inconnu). }\end{array}$ & $\begin{array}{l}\text { Écart entre ce qui est } \\
\text { prévu et ce qui est réalisé } \\
\text { (après vérification auprès } \\
\text { des archéologues } \\
\text { concernés). }\end{array}$ \\
\hline 11 recherches $(6 \%)$ & 20 recherches $(9 \%)$ & $\begin{array}{l}\text { Moins de } 10 \% \text { de projets } \\
\text { permettant d'acquérir de } \\
\text { nouvelles connaissances. }\end{array}$ \\
\hline
\end{tabular}

En effet, très peu de fouilles archéologiques étaient réalisées en 2013 et 2014 dans un cadre de recherche traditionnel (moins de 10 $\%$ ) et un nombre de plus en plus élevé de fouilles se font sans véritable objectif d'acquisition de connaissance. Cela est dû au fait qu'au moment de préparer sa demande de permis, l'archéologue ne sait pas ce qu'il est susceptible de trouver ou, encore, que son mandat consiste tout simplement à dégager les vestiges et à les documenter. Entre la documentation des vestiges et l'acquisition de connaissances, 
il existe un écart considérable que l'archéologue doit combler tant bien que mal lors de la rédaction de son rapport et dans un délai de moins d'un an, selon l'exigence de la loi québécoise.

Il aurait été intéressant de se pencher aussi sur la nature des connaissances acquises au cours des années 2013 et 2014, toutefois les rapports de fouilles n'étaient pas tous disponibles au moment de la rédaction de cet article.

\subsection{Résumé}

En somme, les fouilles sont destinées à plusieurs fins (formation, développement, aménagement, restauration, éducation, mise en valeur, etc.) et la recherche y occupe une place bien mineure. À vrai dire, les fouilles s'insèrent dans des contextes culturel, social, économique et touristique où les enjeux ne concordent pas nécessairement avec la recherche. Elle se fait aisément en milieu universitaire, mais ailleurs elle doit faire valoir sa pertinence durant les fouilles, pour que des analyses puissent être faites et que la diffusion des résultats soit envisagée. De plus, il existe généralement peu de budget pour réaliser des analyses spécialisées, sauf pour faire des datations au radiocarbone ou des analyses zooarchéologiques ou paléoenvironnementales sommaires. N'empêche que certains projets appellent des fonds supplémentaires, lorsque le potentiel et les résultats préliminaires convainquent à la fois l'archéologue et le promoteur de l'intérêt public et de bénéfices éventuels. Plutôt qu'une contrainte de plus à la réalisation de leurs projets, certains promoteurs y voient une valeur culturelle ajoutée, mais ils se font encore rares.

En fait, la protection du patrimoine archéologique prend le dessus sur la recherche scientifique. Ironiquement, l'archéologue n'a reçu aucune formation universitaire en matière de protection du patrimoine archéologique puisque la formation est centrée essentiellement sur la recherche. L'archéologue découvre cette réalité sociale, économique et culturelle en réalisant des interventions archéologiques autres que les chantiers-écoles universitaires. Son apprentissage professionnel se fait avec les firmes et les consultants qui pratiquent dans ce même cadre professionnel depuis maintenant plus de 35 ans. 
Enfin, peut-être faut-il aussi s'interroger sur la pertinence de la protection du patrimoine archéologique si elle n'inclut pas la recherche. D'où la question suivante qui sera centrale à la prochaine discussion : À quoi sert de protéger ce que l'on ne prend pas le temps de connaître ? Si le réflexe actuel consiste à consigner des données, à documenter, puis à conserver le patrimoine archéologique, comment concilier cet exercice avec le processus d'acquisition et la diffusion des connaissances pourtant essentiels pour alimenter la réflexion sur le devenir de la société ? Autre interrogation : qu'advient-il des données et des collections recueillies au fil des années s'il n'existe aucun mécanisme pour les étudier et diffuser les résultats?

\section{Discussion}

L'évolution de la pratique archéologique actuelle sur le terrain a de sérieuses répercussions sur la recherche telle qu'elle a été conçue et structurée traditionnellement, c'est-à-dire en fonction de l'acquisition et de la diffusion des connaissances. Alors que le fondement de la recherche consiste à recueillir, analyser, interpréter les données et publier les résultats en vue de la transmission des connaissances pour le bénéfice de la société, il est beaucoup plus difficile d'y arriver aujourd'hui à cause des enjeux culturels, sociaux et économiques entourant la protection du patrimoine archéologique. Le portrait de la situation n'est pas facile à faire puisque plusieurs éléments doivent être considérés dont les conditions légales et contractuelles qui balisent le travail de l'archéologue.

À la lumière des observations proposées jusqu'à présent, des constats sont établis ici en fonction de ce qui se fait comme recherche sur le terrain, en laboratoire et sur ce qui est diffusé (figure 5). 
Figure 5 : Constats face à la situation actuelle de la recherche archéologique au Québec.

\begin{tabular}{|c|c|c|}
\hline \multicolumn{3}{|c|}{ Constats entourant la recherche archéologique au Québec } \\
\hline & Constat & Principal acteur \\
\hline 1. & $\begin{array}{l}\text { La recherche archéologique reste en plan face } \\
\text { à la protection du patrimoine archéologique. }\end{array}$ & $\begin{array}{l}\text { Gouvernement } \\
\text { (législation) }\end{array}$ \\
\hline 2. & $\begin{array}{l}\text { L'intégration de l'archéologie dans la } \\
\text { planification d'un projet demeure ardue en } \\
\text { amont comme en aval. }\end{array}$ & $\begin{array}{l}\text { Promoteur public ou } \\
\text { privé }\end{array}$ \\
\hline 3. & $\begin{array}{l}\text { L'interprétation se fait sur des bases } \\
\text { historiques plutôt que sur des contextes } \\
\text { archéologiques. }\end{array}$ & Archéologue \\
\hline 4. & $\begin{array}{l}\text { La méthodologie prend souvent le dessus sur } \\
\text { l'interprétation (objectivité vs subjectivité). }\end{array}$ & Chercheur universitaire \\
\hline 5. & $\begin{array}{l}\text { La diffusion se fait par des rapports de terrain } \\
\text { et par des collectifs d'auteurs. }\end{array}$ & Archéologue \\
\hline 6. & $\begin{array}{l}\text { La pratique de terrain est de plus en plus } \\
\text { technique. }\end{array}$ & Archéologue \\
\hline 7. & $\begin{array}{l}\text { Les attentes face à l'archéologue sont } \\
\text { considérables. }\end{array}$ & Société \\
\hline
\end{tabular}

Premier constat (ou évidence !), les interventions sur le terrain ne sont pas destinées à la recherche. La pratique actuelle, encouragée par le cadre légal fédéral et provincial, se limite généralement à récupérer les données sur le patrimoine archéologique avant sa destruction imminente face au développement du territoire. Alors que le Québec considère que toute intervention sur le terrain constitue une recherche archéologique, l'Ontario les qualifie plus justement de travaux archéologiques. De son côté, l'INRAP en France englobe l'ensemble des interventions archéologiques dans une approche préventive où il fait une distinction entre le diagnostic et la fouille, tout en rappelant qu'ils doivent tous deux aboutir à l'acquisition et à la diffusion des connaissances. L'INRAP n'échappe cependant pas au fait d'accumuler des données et des collections sans trop savoir à quelles fins elles sont destinées, comme c'est le cas en Ontario et au Québec d'ailleurs.

Deuxième constat, la pratique archéologique sur le terrain est de plus en plus segmentée en fonction des projets et des promoteurs publics et privés. Les interventions sont dictées par la construction d'un bâtiment, l'aménagement d'un sentier, la restauration d'un bâtiment historique, mais l'intégrité même du site archéologique est 
rarement prise en considération. Alors que le nombre de promoteurs s'accroit et les enjeux de société sont de plus en plus diversifiés, il devient même difficile de faire valoir la conservation du patrimoine archéologique in situ. La restauration du couvent des Augustines à Québec illustre bien le problème: alors que la conservation du patrimoine archéologique était une condition légale de l'autorisation des travaux, les coûts rattachés aux découvertes imprévues étaient tels qu'ils ont mis en péril la réalisation du projet. L'archéologie entre encore difficilement dans la planification des projets et les mécanismes légaux prévus demeurent encore insuffisants, aussi bien en amont qu'en aval.

Troisième constat, en lieu et place de l'étude du contexte archéologique révélé lors des fouilles, on se sert des repères historiques pour y greffer les données archéologiques, ce qui devient un complément à l'histoire déjà connue et non une reconstitution originale de l'histoire du lieu et des modes de vie de ses occupants. Alors que la pratique archéologique s'est affranchie de la science des antiquaires au $19^{\mathrm{e}}$ siècle (DESROSIERS, 2011, p. 21), il ne faudrait pas que les contraintes actuelles, bien que multiples, empêchent les archéologues d'étudier et de reconstituer les contextes dans lesquels les vestiges ont été découverts. C'est l'essence même de leur contribution à la société.

Quatrième constat, la recherche universitaire mise beaucoup sur les laboratoires et les équipements de plus en plus sophistiqués qui peuvent être utilisés par les chercheurs. Il en résulte des avancées significatives en archéométrie et en études environnementales, notamment. Un peu à l'image de ce qui se déroule sur le terrain où l'étude du contexte est trop souvent laissée pour compte, en milieu universitaire c'est l'interprétation qui semble être le talon d'Achille des chercheurs. Les nouvelles technologies les accaparent beaucoup et ils ne disposent plus d'assez de temps pour se consacrer à l'essence même de la recherche archéologique : l'interprétation. De plus, les besoins de rentabilité des laboratoires ralentissent la production scientifique. Les chercheurs eux-mêmes vivent des situations qui les éloignent de la recherche archéologique fondamentale. Il est beaucoup plus aisé d'élaborer des méthodologies complexes et sophistiquées qui ne s'adressent généralement qu'à quelques spécialistes plutôt que d'étudier un site, de révéler sa signification, de 
recevoir des critiques du milieu professionnel et de répondre aux attentes du public.

Cinquième constat, la rédaction d'un rapport au terme d'un an comme l'exige la loi québécoise ne peut se substituer à une publication des résultats de la recherche. Le rapport a pour objectif de décrire ce qui s'est passé et de consigner les données observées ; parfois des interprétations préliminaires y sont avancées, ainsi que des recommandations en vue de la poursuite de la recherche ${ }^{22}$. Diffusé ou non, le rapport reste un document technique. Cela se traduit - au Québec comme ailleurs - dans des rapports techniques qui présentent des résultats uniformes (plats) qui couvrent l'essentiel des données descriptives : le nombre de mètres carrés fouillés, les relevés stratigraphiques, l'inventaire des artefacts etc. De plus, les résultats n'atteignent pas les objectifs de base entourant la recherche archéologique : «... lack the kind of data synthesis needed to describe one of the fundamental aspects of archaeological study: understanding past behavior» (BERGMAN and DOERSHUK, 2003, p. 88). Les publications scientifiques vont évidemment beaucoup plus loin, mais elles se font assez rares. Les publications-synthèses de collectifs d'auteurs tels qu'Air (PINTAL et al., 2015) et Fragments d'humanité (POTHIER, 2016) remédient éloquemment aux lacunes actuelles, mais elles ne devraient pas s'y substituer complètement. À la lumière de la situation actuelle où les collectifs d'auteurs et les diffusions web préconisées par les instances municipales ont la cote, il ne faut pas minimiser - peut-être même revaloriser - l'importance des publications scientifiques.

Sixième constat, une fatigue apparente s'installe dans le milieu professionnel où l'autonomie pour réaliser un travail satisfaisant est de plus en plus rare. À part les contraintes légales, il y a aussi les contraintes contractuelles et la logistique de terrain qui deviennent de plus en plus ardues pour l'archéologue. Il existe aussi une hiérarchie associée au travail de terrain, où des diplômés en archéologie font office de «techniciens », un terme décrié par tous ceux et celles concernées par cette appellation réductrice. C'est une

22 Recommandations qui sont souvent laissées pour compte. 
situation alarmante et préoccupante pour l'avenir de la profession au Québec.

Septième et dernier constat, il existe tout un système influençant la pratique archéologique au Québec et qui fait subir d'énormes pressions aux archéologues. Les dispositions légales et contractuelles, parfois contradictoires entre elles ou encore mal comprises, en découragent plusieurs à vivre de la profession. S'ajoutent à cela les attentes des promoteurs qui veulent maximiser leur investissement dans un marché concurrentiel où l'offre archéologique est beaucoup plus importante que la demande. Leurs attentes peuvent aussi inclure bon nombre d'activités autres que les interventions de terrain, qui s'ajoutent au travail premier de l'archéologue sans nécessairement que ces activités complémentaires soient rémunérées. Bref, le travail en milieu culturel oblige à beaucoup de bénévolat pour les passionnés d'archéologie!

\section{Conclusion}

La recherche archéologique occupe-t-elle encore une place importante en 2016 au Québec ? À la lumière des constats qui viennent d'être faits, on peut dire qu'elle a perdu sa place au soleil. Faut-il s'en inquiéter ? Aux yeux des archéologues, la réponse est évidemment oui, mais ce n'est pas nécessairement le cas pour les autres acteurs qui réduisent trop souvent le rôle de l'archéologue à protéger le patrimoine archéologique plutôt qu'à l'étudier et le comprendre. En ce sens, il apparaît évident que l'État doit réaffirmer son importance en matière de recherche archéologique. L'actuel Règlement sur la recherche archéologique la réduit à des gestes de nature archéologique posés sur le terrain que ce soit pour conserver, détruire, sensibiliser le public, former des étudiants ou, bien sûr, faire de la recherche. Ces gestes n'accordent évidemment pas une place centrale à l'acquisition et à la diffusion des connaissances. Il faudrait donc réviser ce règlement en conséquence, action somme toute assez 
simple puisqu'elle relève d'une volonté ministérielle plutôt que d'une loi gouvernementale.

Une autre évidence se dessine aussi : il devient de plus en plus difficile de mener une recherche archéologique à terme. Tout conjure pour faire dévier l'archéologue de son objectif premier. En premier lieu, c'est la législation qui met l'accent sur la protection du patrimoine archéologique plutôt que la recherche. Cela se justifie aisément aux yeux de la société, ne faut-il pas éviter de détruire les vestiges tangibles de notre mémoire collective sans les documenter ? Mais qu'est-ce qu'on sauve exactement ? Essentiellement, on conserve des données transposées dans des rapports techniques et des collections qui s'accumulent dans les réserves d'archéologie et on repousse encore plus loin le jour où toutes ces données et collections prendront véritablement un sens pour l'archéologue et ultimement pour la société. Cette façon de faire serait sans doute plus légitime si des mécanismes étaient en place pour prévoir et planifier ce que la société veut faire avec tout ce bagage inexploité depuis plusieurs décennies.

Il y a aussi ce qu'on pourrait appeler tout le théâtre autour de l'archéologie : le besoin de la faire connaître, de sensibiliser le public à son importance, de le faire participer au travail de l'archéologue et de lui faire découvrir le passé autour d'eux. Ce sont toutes des actions méritoires, même nécessaires pour que l'archéologie prenne ancrage dans la société québécoise et il reste encore beaucoup à faire à cet égard. Sauf que l'essentiel y est laissé pour compte : la recherche. L'image du diplômé en archéologie qui sert de main d'œuvre (technicien) sur un chantier de fouilles vient en tête et illustre bien le fait que l'archéologue serait mieux utilisé dans la société si on lui permettait de mettre son expertise au service de la connaissance.

Un réflexe social s'implante graduellement et permet aujourd'hui de mieux intégrer la protection du patrimoine archéologique au développement. À cet égard, des progrès notables ont été observés ; avec les années, l'archéologue parvient bien à faire valoir son rôle auprès des promoteurs. Toutefois pour que le réflexe soit socialement plus ancré, il serait nécessaire que l'État reconnaisse davantage le rôle de l'archéologue en faisant de l'adhésion à la communauté professionnelle, l'Association des archéologues du 
Québec et à son code d'éthique, une condition essentielle à l'obtention d'un permis de recherche archéologique.

La formation universitaire en archéologie demeure pertinente en 2016 ; elle fait valoir une démarche intellectuelle complète autour de la recherche sur le plan théorique et pratique. Toutefois pour se préparer à la pratique professionnelle, il n'existe presque rien; il faut donc parfaire ses études en gestion de projets, en administration, en muséologie. Le choc est considérable pour la relève. Peut-on souhaiter qu'une réflexion se fasse au sein de la communauté des archéologues (chercheurs, académiciens et professionnels) pour mieux comprendre les enjeux de société autour de la pratique archéologique, définir plus clairement le rôle de l'archéologue et, conséquemment, contribuer davantage à bâtir son avenir ?

Enfin, faut-il réitérer que peu de recherches archéologiques sont complétées, puis publiées au Québec ? Il ne faut pas oublier que la réflexion de l'archéologue sur la société vue à travers le prisme du site étudié - ou il peut «prendre la mesure des ombres » (MOUSSETTE, 2009) $)^{23}$ - mérite aussi sa place au soleil.

\section{Bibliographie}

BERGMAN, Christopher A. and DOERSHUK F., John. Cultural Resource Management and the Business of Archaeology. In Larry J. Zimmerman, Karen D. Vitelli \& Julie Hollowel-Zimmer (eds), Ethical Issues in Archaeology. Walnut Creek CA, (USA): Altamira Press, 2003, p. 85-97.

BIBEAU, Pierre, DENTON, David \& BURROUGHS André. Ce que la rivière nous procurait : archéologie et histoire du réservoir de l'Eastmain 1. Les Presses de l'Université d'Ottawa et le Musée canadien de l'histoire, collection Mercure, 2015.

23 Cette expression vient d'une alliée naturelle de la recherche archéologique, Catherine Fortin, spécialiste en ethnobotanique et poète. 
CHAPDELAINE, Claude (sous la direction de) (2015). MailhotCurran: un village iroquoien du XVIe siècle, Recherches amérindiennes au Québec, Paléo-Québec, n. 35, 2015.

CODE DU PATRIMOINE.

https://www.legifrance.gouv.fr/affichCodeArticle.do?cidTexte=LEGI TEXT000006074236\&idArticle=LEGIARTI000006845683 （version en vigueur depuis le 24 février 2004).

DESROSIERS, Pierre. L'Archéomuséologie. La recherche archéologique entre au musée, Québec: Presses de l'Université Laval, 2011.

DESROSIERS, Pierre. Archéologie et développement durable au Québec. Archéologiques, n. 29, 2016, p. 66-88.

DESROSIERS, P. M. \& J. RAHM. Sivunitsatinnut ilinniapunga : l'archéologie inuite et l'apprentissage. Études/Inuit/Studies vol. 39, n. 2, p. 259-283, 2015

MOUSSETTE, Marcel. Prendre la mesure des ombres. Archéologie du Rocher de la Chapelle. Île aux Oies (Québec). Québec: Les Éditions GID, 2009.

MOUSSETTE, Marcel \& A. WASELKOV Gregory. Archéologie de l'Amérique coloniale française. Montréal: Lévesque éditeur, 2013.

PARCS CANADA. Investissement dans l'infrastructure. Se préparer au plus gros investissement de tous les temps dans l'infrastructure de Parcs Canada! 2014. http://www.pc.gc.ca/fra/agen/infrastructure.aspX

PINTAL, Jean-Yves, PROVENCHER Jean \& PIÉDALUE Gisèle. Air. Archéologie du Québec. Territoire et peuplement. Montréal: Pointe-à-Callière, musée d'archéologie et d'histoire de Montréal \& Les Éditions de l'Homme, 2015.

PLOURDE, Michel. L'exploitation du phoque à l'embouchure du Saguenay par les Iroquoiens de 1000 à 1534. Ottawa: Collection Mercure, Les Presses de l'Université d'Ottawa et le Musée canadien de l'histoire, 2013. 
POTHIER, Louise. Fragments d'humanité. Pièces de collections. Montréal: Pointe-à-Callière, musée d'archéologie et d'histoire de Montréal et Les Éditions de l'Homme, 2016.

SIMONEAU, Daniel. Îlot des Palais. Rapport de fouilles archéologiques réalisées par la Ville de Québec. Saisons 2006 et 2007. Québec: Cahiers d'archéologie du CÉLAT, n 40, 2014.

WYLIE, Alison. On Ethics. In Larry J. Zimmerman, Karen D. Vitelli \& Julie Hollowel-Zimmer (eds) Ethical Issues in Archaeology. Altamira Press, Walnut Creek CA, (USA): 2003, p. 3-16.

RECEBIDO EM: 01/10/2017 APROVADO EM: 10/12/2017 